\title{
A data processing system for haematology
}

\author{
D. G. CHALMERS \\ From Addenbrooke's Hospital, Cambridge
}

Before considering the use of data processing it is appropriate to define the function of the haematology laboratory within the hospital complex. The main function of that laboratory must be the provision of a system to define the haematological state of the patient. The second important function following on the first is the use of the haematologist's special skills in interpreting data presented and initiating further investigative procedures to establish the diagnosis. The first task, that of measuring accurately the haematological parameters of the patients, is very similar to the work of the biochemist. Almost all patients admitted to our group of hospitals have a routine blood count carried out on admission and in my opinion the laboratory must be organized in such a way that this is carried out with the minimum possible delay; whether this is carried out in association with the biochemists in a specially designed screening laboratory or is retained within the haematology laboratory itself is a matter for discussion, but a system must be devised whereby information is returned to the clinician in charge of the case as quickly as possible. The second type of service, consisting of interpretation and further, more sophisticated investigation, raises different problems and I propose to discuss these two aspects of the haematological service separately.

\section{HAEMATOLOGICAL SCREENING}

The last two years have seen the introduction of the SMA-4 which measures four parameters on whole blood and the promise of the Coulter-S which carries out a similar function. These pieces of equipment process samples at the rate of approximately one sample per minute and in a large laboratory complex are probably best serviced by an on-line control system. In the situation, however, where on-line facilities are not available, unless the original output of the machine is to be used, the transcription of four parameters in a form acceptable to the clinician throws a considerable clerical load on the average laboratory. To this must be added the distinct problem of marrying the results to the correct source. Processing samples in batches, whether simultaneously or sequentially, allows the use of statistical procedures for quality control, and it is important that any data processing system should take account of this need.

\section{INTERPRETATION AND FURTHER INVESTIGATION}

Examples I would give of this type of work are the examination of stained films, preparation of LE cells, serological titrations, and coagulation investigations. All of these require considerable technical skill. The majority of them must be carried out manually and the result determined visually and reported either in a descriptive form or in some type of quantitative measurement. Although automation may play some part in these test systems, their complexity suggests that they will remain manual tests for some time to come.

The efficiency of the hospital unit in relation to the care of the patient can be enhanced by the haematologist's ability to detect changes in individual patient's results as they pass through the laboratory, either by initiating further investigations or by making constructive comments to the clinicians which help in the day-to-day management. In order to carry out this function properly it is necessary for the haematologist to have available previous results on the patient. This allows him to make interpretations on the basis of information provided from his own laboratory but in no way supersedes the patient's clinical record which should, in itself, be comprehensive. Bearing these factors in mind and in the knowledge that an on-line system was unlikely to be available before 1972, if then, I have, with the aid of expert advice from Cambridge Computers Services Ltd, created on paper a comprehensive, off-line, updated, cumulative patient data processing system for haematology.

The reasons for limiting this to haematology only are numerous. First, experience has been gained elsewhere of systems entirely designed for biochemical purposes. Although it is possible to graft haematological work on to these systems it seemed appropriate to start with haematology and see how comprehensive a system devised for this purpose might be and whether it would be possible to add to it the other aspects of clinical pathology at a later 
date. Secondly, the introduction of any system strains the endurance of the laboratory staff to breaking point and inevitably decisions, sometimes radical, have to be made in order to ensure that the system functions properly. If a system is confined to one discipline, it is possible for the senior person to make this decision without consultation with his colleagues. There are also a number of other personal and local reasons for the decision.

At the time I accepted the invitation to speak at this symposium I hoped that funds would become available which would have allowed us to carry out a feasibility study and to produce some factual information. I regret to say that my application has not found favour with the relevant authorities and money for the development of this system has not been forthcoming. I was led to believe, however, that it was not to be supported because there were rather similar schemes from which information would shortly become available. I have to report that efforts to locate these other schemes have failed and I therefore offer my system in the knowledge that although it is untried at least it appears to have no serious competitor.

\section{THE SYSTEM}

PATIENT AND SAMPLE IDENTIFICATION We have been much concerned with the considerable amount of transcription necessary when an investigation is required on a sample taken from a patient. For this reason we have decided to adopt the method described by several authors and used by Professor Whitehead, where punch cards containing the patient's identification are issued to the wards for use as request cards. In a hospital with a computerized records system these cards would be a byproduct of that system. As our computer is still in the planning stage the laboratory would, of necessity, have to create this information for each patient as a result of information given to us by the records department. Unfortunately this will increase our punching costs considerably. The punch cards to use would be those which incorporate specimen identification tags. There are available small tagged cards punched with a number identical to a number punched in the master 80column punch card which contains the patient's identification (Fig. 1). This tag is attached to the specimen and it is possible manually or by machine to marry card and specimen exactly, transcription on to the specimen label being eliminated (Fig. 2). All request cards and samples coming to the laboratory would be married without difficulty. The patient's request cards would be sorted manually into types of tests. It is proposed to introduce a Technicon sampler 40, linked to the SMA-4, which would automatically identify and print the sample number at the time the readings were being made. The normal output of the SMA-4 in the form of a graph would be retained for monitoring purposes and for immediate return of urgent information to the wards. In

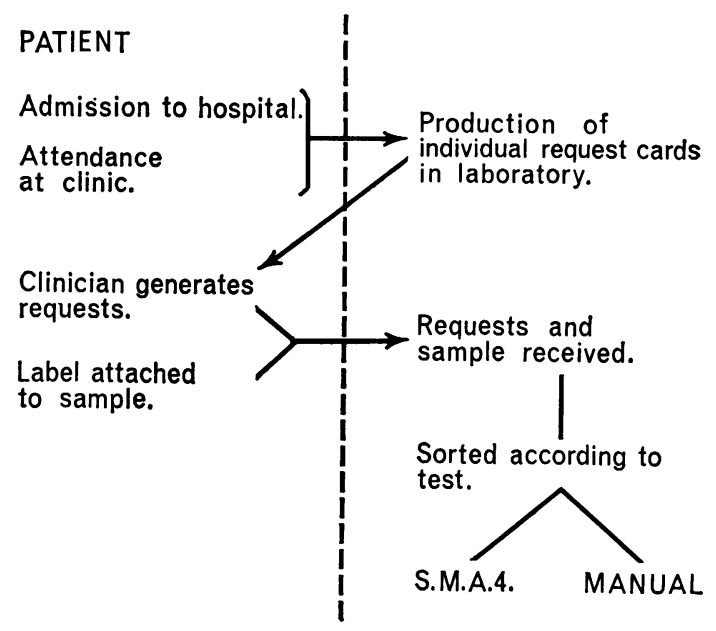

FIG. 1. Specimen identification exchanges between hospital and laboratory.

addition, however, it is proposed by means of an analog digital converter and tape punch to produce on paper tape the results of the batch. The patient's cards relating to this batch and the tape containing the results, also identified by specimen number, would be sent to the computer centre for processing (Fig. 3).

In order to produce the 80 -column request cards punched with the patient's identification and information, it will be necessary to have in the laboratory a sophisticated card punch, which will also be used for the production of work sheets. It should be noted that the introduction of the Sampler 40 and the punch tape output to the SMA-4 results in the transcription, automatically, of some $80 \%$ of the total data generated in the laboratory.

All the remaining data must, of necessity, be produced manually and subsequently transcribed into machinesensible form. For this purpose each batch of cards relating to one test is fed into the card punch machine and with suitable programming an appropriate work sheet is created (Fig. 4) and is given to the technician who sets up the samples in the order laid down on the work sheet and subsequently transcribes the results into the appropriate spaces in a preagreed numerical system. Obviously in the majority of serological and coagulation procedures it is easy to see how a numerical answer may be given. It is perhaps more difficult to interpret the appearance of the stained film in this manner. However, over the last two years we have had experience of a reporting system in which the appearances of the stained films are recorded by a numerical method. As the information obtained is to be processed by a computer, we visualize no difficulty in the use of symbols which in fact will cover not only the various film abnormalities which are encountered but which will allow introduction of new variables if required. When the work lists are completed they are returned to the card punch operator who feeds in the relevant cards in the correct order and is 


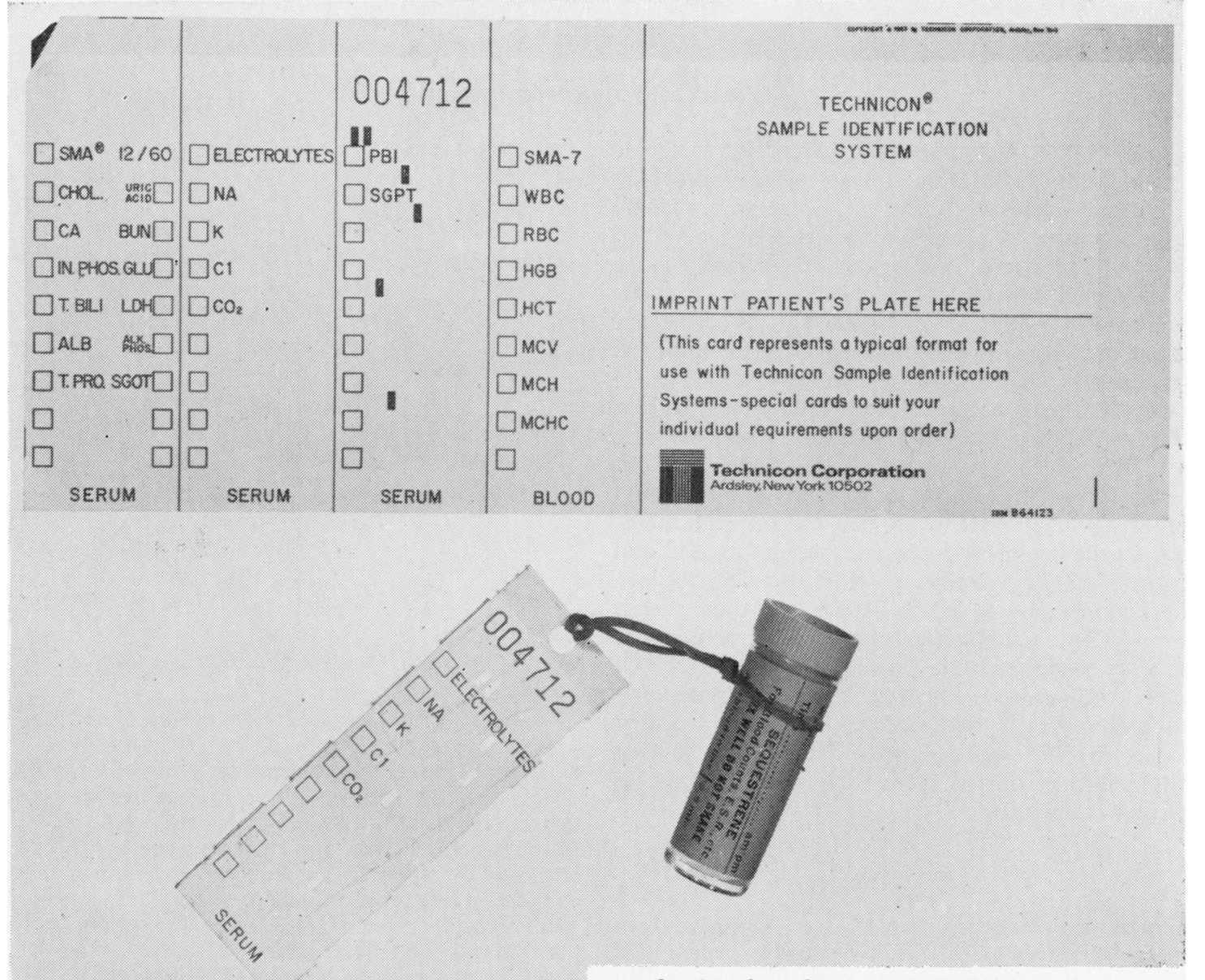

FIG. 2. Punch card and sample identification tag.

\section{MANUAL:}

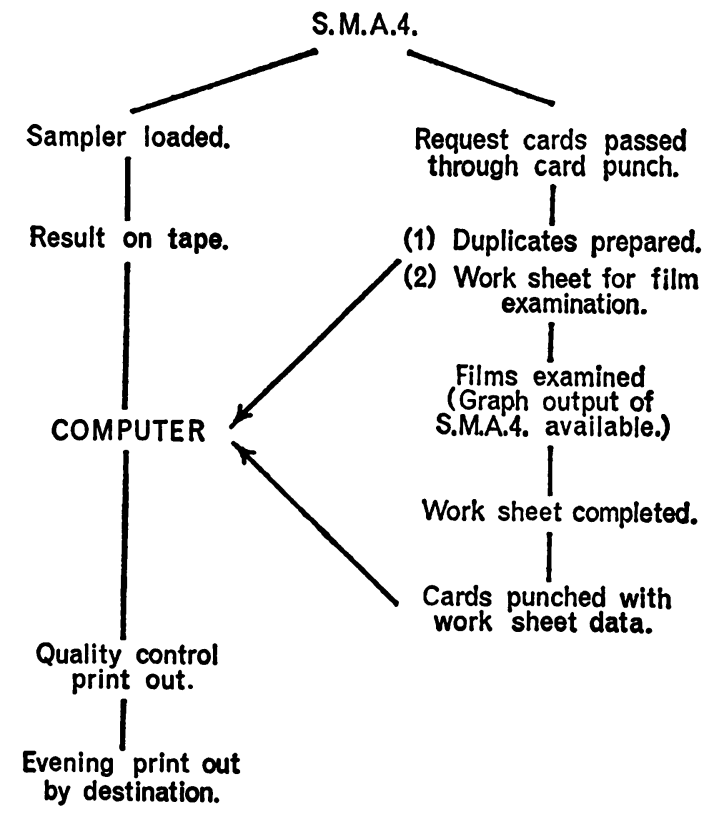

FIG. 3.

Request cards and samples sorted by test.
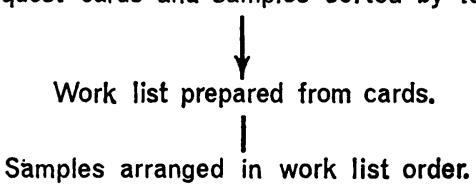

FIG. 4. Evening print out by destination.

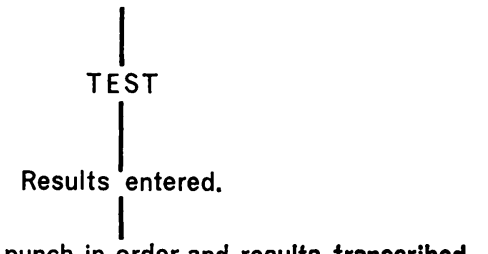

Cards fed through punch in order and results transcribed.

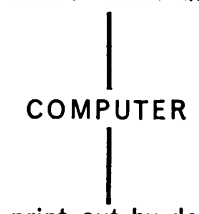

FIG. 3. Handling of specimens and information to the SMA-4.

FIG. 4. System for handling manually performed tests. 
Previous days results placed in identification order.

Disc file searched for further information about each patient.

PRESENT

Information added to file.

All information printed.

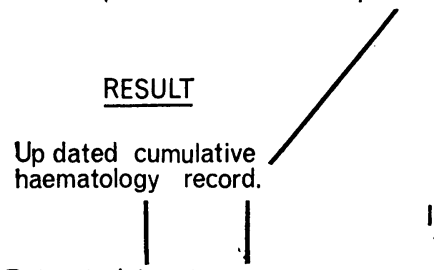

Returnto laboratory.

Scrutiny by pathologist.

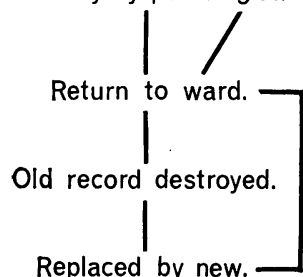

FIG. 5. Function of the computer during morning run.

only required to punch in the new data. The cards are then ready to be sent to the computer centre.

It is proposed that the information required should be processed by the computer at two periods each day. Each evening all results obtained during the day on either punch cards or paper punch tape would be fed into the computer. These would be reorganized in the order of destination and results for each patient on a particular ward would be issued on a single sheet of paper containing the patients' names, their identification numbers, and the results obtained that day. The following morning a second run is carried out (Fig. 5) which will include those results reported the previous day and any results obtained in the interim. During this time the computer will search a disc file containing all results obtained over the preceding two months and stored under the patient's identification. This disc file will be updated with the latest test information obtained. At the same time all those patients to whose file new information has been added will have their complete updated haematological record produced in the printed form. This sheet, after scrutiny by the haematologist, will be inserted in the patient's clinical notes taking the place of the previous sheet. In this way the patient's entire haematological information is presented in a cumulative updated fashion, reducing the amount of paper containing the information to a minimum.

\section{A. DISC FILE SEARCHED}

(1) All records not containing information less than 2 months old removed.

\section{B. MASTER MAGNETIC TAPE SEARCHED}

(1) Old disc records inserted.

(2) Tape searched for information on newly aquired patients which is removed.

\section{DISC FILE UPDATED WITH NEW INFORMATION (B.2.)}

FIG. 6. Function performed during search procedure.

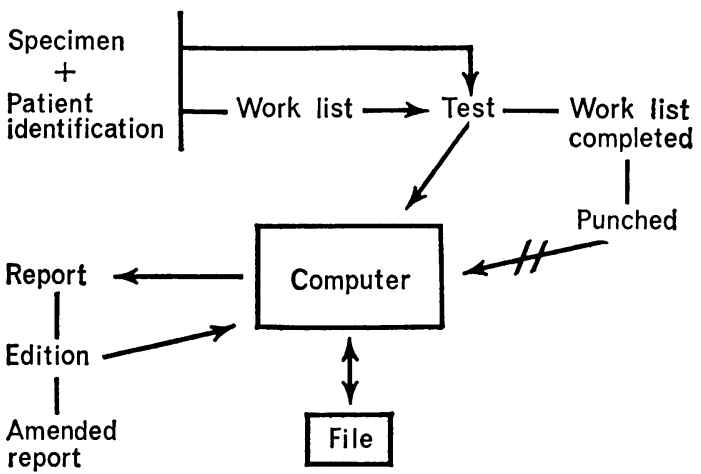

FIG. 7. Diagram of information flow in an off-line system.

At regular intervals, the disc file containing the patient's information will be sorted again (Fig. 6). The records of those patients on whom no further information has been collected during the previous two months will be transferred to a permanent magnetic tape store. At the same time the magnetic tape will be searched for information on those patients who have appeared on the disc file during the previous week. In this way it is possible to maintain all the information about tests performed in the laboratory in a form which can be utilized for many purposes in the future and to keep information of current value in a rapidly accessible form on disc file. During the evening run, in addition to producing the ward sheet, the computer will carry out quality control analysis on tests carried out that day. It is proposed to use the information in the store for other statistical purposes, which would include the determination of normal values for different age groups and, of course, valuable information on costing.

\section{COMMENT}

There are certain features of this scheme which I think should be emphasized (Fig. 7). So that all relevant test information and comment may be kept in the file, it is necessary to abandon the everyday language used for comment and to substitute for it 
a stylized, computer-sensible alternative. Although imposing an unwelcome discipline on the poetically minded, it does induce uniformity and clarity of expression.

Secondly an important point is that all information obtained in the system is available in readable form and it is possible to intervene manually to obtain a particular result during processing in the laboratory or, in the event of a computer breakdown, to produce the information in manual form.

Thirdly, the effect on the technical working of the laboratory is almost entirely advantageous, although rigid uniformity is imposed on the reporting of peripheral blood films. The positive advantages are the reduced amount of clerical work and the production of preprinted work sheets for technical use superseding the present hand-written work sheet. The use of numbered punched labels as opposed to the present system of printed or hand-written labels should have little adverse effect, as almost all specimens for serological tests are given laboratory batch numbers before the tests are instituted.

RUNNING COSTS No scheme of this kind can be contemplated unless a realistic estimate is available of running costs which may be compared with present costs. A certain amount of capital equipment is required. It has been estimated that in the average year some 12 million characters of information have to be generated in the laboratory. By producing in bulk individual request cards for each patient and machine-sensible data from the SMA-4 it is possible to reduce this to some 2 million characters in a year. To do this, however, it is necessary to invest first of all in a sophisticated card punch, such as that produced by IBM. The SMA-4 is modified first by the introduction of the Technicon 40 sampler, which automatically reads the sample number card, and of a peak picker, an analog digital converter and paper tape punch. The total cost of this equipment is approximately $£ 10,000$. Although laboratory technical and clerical staff can learn to use the card punch with moderate proficiency, the amount of punching required will justify the employment of a whole-time punch card operator. As the clerical staff are relieved of a vast majority of simple clerical and filing duties it may be possible to compensate for this appointment by reducing the number of general clerical staff. This project has not yet undergone a feasibility study during which the scope and depth of the project would be assessed and the detailed pattern of progress of the information through the laboratory determined. The cost of this feasibility study would be some $£ 600$. In addition, there would be a programming cost divided into those programs necessary for the production of the results and the storage of information and subsequent programs which would be related to the acquisition of statistical data and costing information.

Finally there is the rental of the computer. Because the computer is required for six days a week it is necessary to make use of a commercial computer bureau which can guarantee such a service. Using a moderately high-speed computer with disc packs, the total daily running time has been estimated at 45 minutes, the cost of which on a contract basis is $£ 35$ per hour.

It has not been possible to cost our present manually operated cumulative filing and reporting system accurately. The amount of technical time involved is considerable, but, ignoring this, the cost of providing request cards, cumulative report cards, photocopying, and filing is in the region of $£ 2,500$. We are therefore proposing a general increase in cost for what appears, superficially, the production of the same amount of information. However, we are introducing, first, a system which reduces the amount of clerical work required by technical staff; secondly, a system which is capable of considerable expansion to a degree which would be impossible by our present manually operated system; thirdly the production of a system whereby information once obtained can be used for quality control purposes, and for the establishment of normal values in different age groups and different sections of the population, so contributing more information to the clinician.

There are those of us who feel that our main effort in recent years has been to provide an everincreasing amount of information with the same, or at best, slightly increased financial resources but there must be few who can confidently state that the standard of information so produced has improved. When one sees large amounts of money being allocated to projects which can benefit only a very small section of the community, is it unreasonable to ask for additional funds which will contribute appreciably to the standard of care of all hospital patients?

\section{FUTURE DEVELOPMENT}

Having defined the system which is the minimal acceptable within a clinical laboratory I would now like to go on and discuss a more comprehensive system which I think would make sensible use of the latest advances and could well provide interesting information on the interface problem between the medically trained worker and the machine. Basically the overall scheme would remain unaltered (Fig. 8). It is probable that, in future, the generation of 


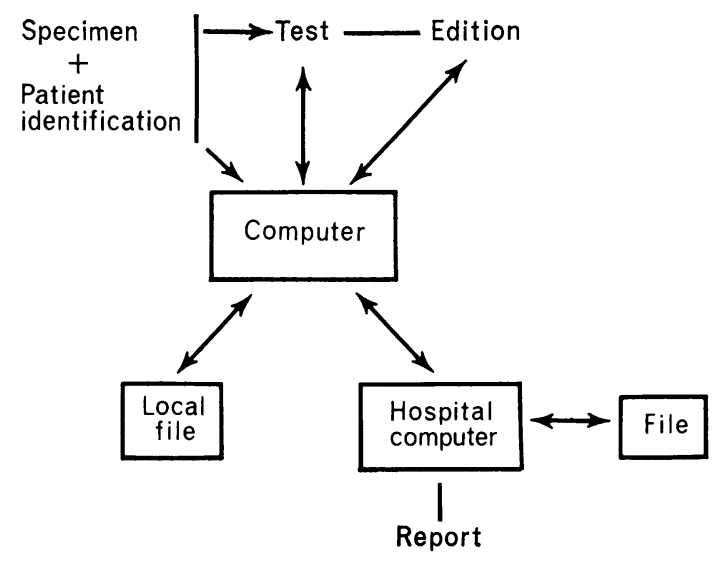

FIG. 8. Diagram of information in an on-line system.

requests will no longer be on the punch card basis but transmitted through the hospital's own computer system. Specimen identification of the form already described, ie, the punched card label, would have to remain, as no development can be foreseen whereby specimens may be poured down a terminal and at laboratory level specimens must be linked with request forms. Instead of producing the information from the SMA-4 in the form of paper tape this machine would now be linked on-line to a computer of moderate size and the data acquired would be in the form described by previous speakers. This represents only a modest advance in the service provided with a considerable increase in cost, and I consider the justification for a computer within the laboratory will lie in its ability to serve a number of terminals within the laboratory complex. The terminals $I$ have in mind would be situated first at the film screening bench and these would probably consist of visual display units. Films would be presented to the technician at the microscope with a batch number marking, which would be fed into the visual display unit which would respond by presenting the patient's name linked to that sample number and the result of the numerical analysis carried out on the SMA-4. The technician would examine the film and then, according to a predetermined code, key in the observations which would automatically be linked to the record in the computer. Whether an extension of this type of peripheral terminal to the other stations at which manual examinations are carried out is worth while is a matter for experiment. Possibly manually entering data on work sheets which are then punched by an experienced operator is a more efficient method of inserting information. However, the use of the visual display unit would certainly be essential for the senior person whose responsibility it was to comment on the abnormal results and to suggest further investigations and possibly diagnosis. This terminal would have to have access not just to the immediate result but to the cumulative updated report which would be held in the computer file. As each film was examined the haematologist would be able to display before him the cumulative record of that particular patient and as a result would key in his observations, many of which would be in a stylized form. A possible but more expensive development in terms of cost would be the reporting of bone marrow findings. It is possible to develop programs using a modified teaching machine or directly using an on-line terminal to devise a system of interrogation whereby a marrow report is created by answers to a series of questions so programmed that each answer determines the next question to be asked. The end result is the creation of a standardized report, but the introduction of a final comment in ordinary language is also possible. This system has been developed in Sweden for radiological interpretations and is perfectly possible for the interpretation of bone marrow smears.

Given reliability of function the system could be extended for use in the blood transfusion department: interrogation and updating of blood grouping files by technicians through a visual display unit has numerous advantages, and a system whereby available blood stocks for the whole hospital and the amount of blood prepared for individual patients would be stored in a file and displayed as required would make many of the present rather laborious procedures in the transfusion laboratory redundant; it could also, by suitable programming, lead to a more efficient use of the rather limited stocks held by individual hospitals.

\section{THE CASE FOR A LOCAL INSTALLATION}

If one visualizes computer development in the laboratories along these lines, taking into account the individual requirements of the other sections of the clinical laboratory, the possibility that the systems being installed at present will be adequate is remote. There are those who would argue that the facilities outlined should be provided by a large central computer. This may be so but many of these functions are of a specialized nature and to search extensive hospital files to capture very limited amounts of information from each file may be an inefficient exercise. In addition the proliferation of terminals required in a laboratory for such a system under almost continual use during the peak periods of the day might in fact throw a strain on a hospital multiple access system. There appears to be a strong case for use of a computer of moderate size within 
the laboratory complex, a satellite of the main hospital computer, and all information which it contained about patients would be transferred to master files in the main computer centre. The laboratory would use its own computer as a multiple access system for on-line input and output.

To many of those working in the laboratory today, trying to cope with ever-increasing work loads with inadequate staff and inadequate accommodation, this concept may seem unrealistic. I would suggest that although it may be unrealistic for most of us at the present time it is the inevitable future. Technology has given us the ability to generate large amounts of information from individual samples obtained from patients. We must acquire methods of handling this information; more important we must devise methods for grading this information as it affects the health of the patient, and the clinician must be informed clearly in those cases where a particular piece of information is considered vital. We can no longer rely on the situation where, by manually scanning a mass of data returned from the laboratory, the clinician is expected to pick out, in the relatively short time at his disposal, the important features. It behoves us to use all the technological advances available to us to capture information, verify it, analyse it, comment on it and present it to the clinician in an easily assimilable form. This is why we should want a computer and this is what we should want to do with it.

\section{SUMMARY}

The pattern of a haematological service in a general hospital is defined and the requirements for a data processing system are stated. A proposed off-line data processing scheme using a commercial bureau is described, and the advantages are discussed in relation to the additional cost. Finally an on-line system is outlined in which not only are results collected directly from the analysing equipment but technical staff have access to information and can enter results directly through visual display units.

\section{Discussion : A data processing system for haematology}

\section{ROBERTSON SMITH}

Dr Chalmers has given us a clear account of a system of data processing as applied to haematology. I am speaking theoretically also, but in Reading we are considering an on-line computer to serve both haematology and biochemistry, initially linked to a combined screening laboratory using an SMA-12 and SMA-4. Both Dr Flynn and Dr Chalmers have given sound reasons for using an on-line system, particularly for the detection of errors and quality control.

In haematology there are two particular problems. One is access to the record store, which for most investigations can be on a once or twice daily basis but for some, particularly grouping and transfusion work, must be instantaneous. The other problem relates to the nature of haematology reports which are a combination of numerical and verbal.

In an open haematology laboratory a large proportion of the requests come from general practitioners. At the Royal Berkshire Hospital they form some $40 \%$ of the requests and the number is increasing. This presents us with one of our biggest problems in relation to patient identification, especially when trying to relate a hospital patient with earlier requests from the general practitioner before the patient had a hospital number. Until there is a universally acceptable unique identification number this problem seems almost insoluble, and I would like to know how others are dealing with this problem.

By far the commonest request is for a single haemoglobin estimation and I wonder whether it is really worth while routinely searching for previous results when so many will yield nothing. We all know the ideal would be for the person initiating the request to indicate whether there had been any previous investigations and only these could then be retrieved, but for various reasons one rarely receives this information.

I agree that most of the work passing through a routine haematology laboratory can be reported in a simple coded form. Taking routine blood counts, prothrombin estimations, simple coagulation tests, blood grouping, and cross matching, there is less than $1 \%$ which will require any long written report and these, together with bone marrow reports could, if necessary, be kept in a small conventional file with simply a reference to them on the computer store. This would, I think, give a simple comprehensive storage.

Emergency work done outside normal laboratory hours may present difficulties unless the record retrieval system can be operated by the technician on duty.

When measured against the enormous volume of requests now pouring through large haematology laboratories, the benefits to laboratory staff and the benefits to the clinical staff, the costs mentioned by Dr Chalmers are indeed trivial. I think the benefit to the clinician in producing easily read reports has not been sufficiently stressed in the past and must be a good selling point. 